\section{THU0416 PEGLOTICASE RESPONSE IMPROVEMENT BY CO-TREATMENT WITH METHOTREXATE: RESULTS FROM THE MIRROR OPEN-LABEL CLINICAL TRIAL IN PATIENTS WITH UNCONTROLLED GOUT}

J. Botson ${ }^{1}$, P. M. Peloso ${ }^{2}$, K. Obermeyer ${ }^{2}$, B. Lamoreaux ${ }^{2}$, M. E. Weinblatt ${ }^{3}$, J. Peterson ${ }^{4} .{ }^{1}$ Orthopedic Physicians Alaska, Anchorage, United States of America; ${ }^{2}$ Horizon Therapeutics plc, Lake Forest, United States of America; ${ }^{3}$ Brigham and Women's Hospital, Boston, United States of America; ${ }^{4}$ Western Washington Arthritis Clinic, Bothell, United States of America

Background: Gout is a painful inflammatory arthritis caused by persistently elevated serum uric acid (sUA) levels. Pegloticase, an infused recombinant PEGylated uricase, rapidly lowers sUA levels by converting uric acid to allantoin, a water-soluble molecule that is readily excreted by the kidneys. In the phase 3 clinical trials, $42 \%$ of patients ${ }^{1}$ dosed with pegloticase every two weeks maintained sUA levels below $6.0 \mathrm{mg} / \mathrm{dL}$ during months 3 and 6 of pegloticase treatment. The loss of pegloticase efficacy has been attributed to the development of anti-drug antibodies (ADAs) ${ }^{1-3}$ and these ADAs have been associated with infusion reactions (IRs). ${ }^{1,2}$ Case reports ${ }^{4}$ and prospective case series ${ }^{5,6}$ indicate that methotrexate (MTX) may allow patients to attain more complete therapeutic benefits, presumably through attenuation of pegloticase immunogenicity. The current study prospectively examines the efficacy and safety of MTX-pegloticase co-therapy in patients with uncontrolled gout.

Objectives: To assess efficacy and safety of concomitant pegloticase and MTX therapy in patients with uncontrolled gout.

Methods: Adult patients with uncontrolled gout who were beginning pegloticase therapy were considered for enrollment in this ongoing multicenter, open-label, efficacy and safety study of pegloticase with MTX co-treatment (NCT03635957). Patients were administered oral MTX $(15 \mathrm{mg} /$ week $)$ and folate $(1 \mathrm{mg} /$ day $)$ 4 weeks prior to the first pegloticase infusion (Day 1) and throughout the pegloticase treatment period. Blood was drawn prior to each infusion to measure sUA level, monitor clinical parameters, and examine for ADA development. All patients followed typical IR prophylaxis protocols (fexofenadine one day before and the morning of each infusion and acetaminophen and IV corticosteroid the morning of each infusion). Patients also received gout flare prophylaxis with either NSAIDs, colchicine or prednisone initiated at least 1 week prior to Day 1 . The primary study outcome was the proportion of responders, defined as sUA $<6 \mathrm{mg} / \mathrm{dL}$ for at least $80 \%$ of the time during month 6 (weeks 20,22, and 24). All analyses were performed on a modified intent-to-treat population, defined as patients who received $\geq 1$ pegloticase infusion.

Results: A total of 17 patients were screened and 14 patients (all men, average age: $49.3 \pm 8.7$ years) were enrolled. On Day 1 , mean sUA was $9.2 \pm 2.5 \mathrm{mg} / \mathrm{dL}$ and 12 of the 14 patients had visible tophi. At the 6 months timepoint, 11/14 $(78.6 \%, 95 \% \mathrm{Cl} 49.2-95.3 \%)$ met the responder definition, with 3 patients discontinuing after meeting stopping rules (pre-infusion sUA values greater than $6 \mathrm{mg} / \mathrm{dL}$ at 2 consecutive scheduled visits). All patients tolerated MTX. One serious AE of bacterial sepsis occurred (resolved). AEs that occurred in $>1$ patient during the co-treatment period were: diarrhea and upper respiratory tract infection in 3 patients each, sinusitis, muscle strain, and hypertension in 2 patients each. Gout flares occurred in 12/14 (85.7\%) patients. No new safety concerns were identified.

Conclusion: An increased proportion of patients maintained therapeutic response at 6 months when treated concomitantly with MTX and pegloticase $(78.6 \%)$ when compared to the previously reported $42 \%$ using pegloticase alone. ${ }^{1}$ These results support and reflect the improved response rates demonstrated in two prior case series. ${ }^{5,6} \mathrm{~A}$ definitive randomized double-blinded trial evaluating pegloticase with MTX vs. pegloticase with placebo is ongoing. References:

[1] Sundy JS et al. JAMA 2011;306:711-20.

[2] Baraf HS et al. J Clin Rheumatol 2014;20:427-32.

[3] Lipsky PE et al. Arthritis Res Ther 2014;16:R60.

[4] Bessen SY et al. Semin Arthritis Rheum 2019;49:56-61.

[5] Botson J, Peterson J. Ann Rheum Dis 2019;78:A1289.

[6] Albert JA et al. Arthritis \& Rheumatology 2019;71(S10):A1236.

Disclosure of Interests: : John Botson Grant/research support from: Horizon Therapeutics (PI and study site), Radius Health (study site), Consultant of: Horizon Therapeutics, Speakers bureau: Celgene, Eli Lilly, Horizon Therapeutics, Mallinckrodt, Novartis, Pfizer, Paul M. Peloso Shareholder of: Horizon Therapeutics, Employee of: Horizon Therapeutics, Katie Obermeyer Shareholder of: Horizon Therapeutics, Employee of: Horizon Therapeutics, Brian LaMoreaux Shareholder of: Horizon Therapeutics, Employee of: Horizon Therapeutics, Michael E. Weinblatt Grant/research support from: BMS, Amgen, Lilly, Crescendo and Sonofi-Regeneron, Consultant of: Horizon Therapeutics, Bristol-Myers Squibb, Amgen, Abbvie, Crescendo, Lilly, Pfizer, Roche, Gilead, Jeff Peterson Grant/research support from: Abbvie, UCB, Smith Klein, Horizon Therapeutics,
Consultant of: Lilly, Norvartis, Horizon Therapeutics, Speakers bureau: Lilly, Novartis, Horizon Therapeutics, Abbvie, Genentech DOI: 10.1136/annrheumdis-2020-eular.3932

\section{THU0417 \\ READMISSION RISK AND QUALITY OF CARE IN PATIENTS PRESENTING TO THE EMERGENCY DEPARTMENT WITH GOUT FLARES}

L. Brunetti ${ }^{1}$, J. Vekaria ${ }^{2}$, P. Lipsky ${ }^{3}$, N. Schlesinger ${ }^{4} .{ }^{1}$ Ernest Mario School of Pharmacy; Rutgers, The State University of New Jersey, Piscataway, United States of America; ${ }^{2}$ Mario School of Pharmacy; Rutgers, The State University of New Jersey, Piscataway, United States of America; ${ }^{3}$ RILITE Research Institute, Charlottesville, VA, United States of America; ${ }^{4}$ Rutgers Robert Wood Johnson Medical School, New Brunswick, United States of America

Background: Gout is the most common form of inflammatory arthritis and its economic burden is substantial, with estimates for the overall cost exceeding $\$ 20$ billion (US) annually. Contributing to the economic burden are hospital admissions and iatrogenic events associated with pharmacotherapy. Identification of modifiable risk factors would be an important contribution to clinical practice.

Objectives: The aim of this study was to identify opportunities for enhancing gout care in patients presenting to the Emergency Department (ED) with gout flares.

Methods: This retrospective cohort study used data from electronic medical records (EMR) at a large community hospital. All consecutive patients visiting the medical center ED with a primary diagnosis of gout from 1/1/2016 to 7/1/2019 were included. Patients were then followed for 90 days to determine whether they were readmitted to the ED for any reason. A chart review identified whether they were on appropriate medications in terms of gout flare management. All data were summarized using descriptive statistics. A multiple logistic regression was constructed to identify risk factors for ED utilization within 90 days of the index visit.

Results: A total of 214 patients were included in the analysis. Most patients were male $(79 \%)$, mean age was $59.4 \pm 15.6$ years, and mean Charlson comorbidity index was $0.5 \pm 1.14$. The most common medications prescribed during the ED visit included NSAIDs (41.6\%), opioids (28\%), corticosteroids $(26.6 \%)$, and colchicine (21\%). Allopurinol and febuxostat were initiated in the ED in $4.7 \%$ and $0.9 \%$, respectively. Discharge medications for the management of gout included NSAIDs $(37 \%)$, corticosteroids $(34.6 \%)$, opioids $(23.8 \%)$, colchicine $(14 \%)$, febuxostat $(7 \%)$, and allopurinol $(6.5 \%)$. Of the patients sent home with an opioid, $40 \%$ were newly prescribed. An anti-inflammatory medication was not prescribed in $29.6 \%$ of patients discharged from the ED. Readmission within 90 days was recorded in $16.8 \%$ of patients. Of these readmissions, $33.3 \%$ were gout-related and $11.1 \%$ were cardiac related.

After adjusting for age and comorbidity index, patients receiving colchicine were 2.8 times more likely $(\mathrm{OR}, 2.81 ; 95 \% \mathrm{Cl}, 1.12$ to $7.02 ; \mathrm{p}=0.027)$ to return to the ED within 90 days. The most common cause of readmission in this subset was gout-related $(54.5 \%)$.

Conclusion: Nearly $30 \%$ of patients were discharged from the ED without an anti-inflammatory medication, whereas initiation of urate lowering therapy was rare. Opiates were used frequently, but the indication was uncertain. Only $5.6 \%$ of subjects revisited the ED for gout-related diagnoses in the subsequent 3 months. Colchicine prescription was associated with an increased risk of gout-related ED utilization within 90 days. Treatment of gout in the ED is sub-optimal and often does not follow established guidelines.

Disclosure of Interests: : Luigi Brunetti Grant/research support from: Astellas Pharma, CSL Behring, Consultant of: Horizon Foundation of New Jersey, Janaki Vekaria: None declared, Peter Lipsky Consultant of: Horizon Therapeutics, Naomi Schlesinger Grant/research support from: Pfizer, AMGEN, Consultant of: Novartis, Horizon Pharma, Selecta Biosciences, Olatec, IFM Therapeutics, Mallinckrodt Pharmaceuticals, Speakers bureau: Takeda, Horizon DOI: 10.1136/annrheumdis-2020-eular.3302

\section{THU0418}

CAROTID ATHEROSCLEROSIS AND SONOGRAPHIC SIGNS OF URATE CRYSTAL DEPOSITS IN GOUT: AN ASSOCIATION STUDY

1. Calabuig ${ }^{1}$, A. Martínez-Sanchis ${ }^{1}$, M. Andrés ${ }^{1,2} .{ }^{1}$ Hospital General Universitario de Alicante-ISABIAL, Sección de Reumatología, Alicante, Spain; ${ }^{2}$ Universidad Miguel Hernández, Medicina Clínica, Alicante, Spain

Background: Carotid subclinical atherosclerosis is prevalent in patients with gout, although poorly predicted by cardiovascular risk assessment tools. Gout itself is deemed to contribute to its development. However, a previous report did not show an association between clinical characteristics of gout and the presence of subclinical atherosclerosis [1] 